\title{
¿Nuevas drogas en el adolescente?
}

\section{New drugs in the teenager?}

Oscar Sánchez-Guerrero, ${ }^{1}$ Sergio I Muñoz-Fernández ${ }^{2}$
En la actualidad, para el ser humano es cada vez más variada la posibilidad de utilizar sustancias novedosas con un efecto en el organismo en general y el sistema nervioso central en particular, que le llevan a experimentar estados de bienestar, de expansión, de omnipotencia y otros.

Los jóvenes experimentan una serie de cambios estructurales (en el cerebro) y sociales (la comprensión del mundo debido a los cambios referidos), por lo que estas sustancias y sus efectos son altamente valorados como parte de sus procesos de llegada al mundo adulto.

Hoy día, con la difusión de la información de manera explosiva e incontrolada, entre los jóvenes se difunden más fácilmente las posibilidades de sensaciones novedosas con sustancias que se van sintetizando frecuentemente y de las que aún no conocemos del todo sus efectos, pero que rápidamente son superadas por otras aún más novedosas y desconocidas.

Esto nos lleva a la coexistencia de las Ilamadas "nuevas drogas" con las "viejas drogas" y de las que se supone conocemos todo o al menos mucho más, y, sobre todo, que se hayan vuelto una especie de terreno conocido por los jóvenes para que su consumo no implique misterios, deseos, fantasías. Sin embargo, es necesario dejar claro que esas viejas drogas siguen siendo un problema mayor para el desarrollo de las personas y que sus efectos son, sin duda, mortales para la maduración de quienes las consumen. ${ }^{1}$ La evidencia nos muestra que el alcohol, la mariguana y su consumo combinado son las sustancias que mayores riesgos conllevan para el desarrollo de los jóvenes, ${ }^{2}$ siempre en función de los eventos vitales de cada sujeto, sus situaciones de riesgo (es decir, la coexistencia de otros diagnósticos psiquiátricos, como el trastorno de atención) ${ }^{3} y$, en fin, el ambiente familiar del que provienen. ${ }^{4}$

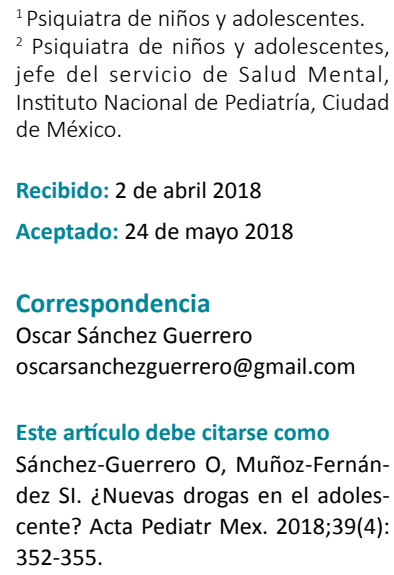


Cuando hablamos de drogas debemos dejar claro que la aproximación debe ser médica, para eliminar connotaciones morales o de juicio de quienes las consumen; entender esto como un problema médico permitirá ser más objetivos en las definiciones y en la comprensión de la problemática a fin de llegar a las posibilidades terapéuticas cuando esto sea posible.

En el consumo de sustancias tóxicas se consideran tres aspectos (eliminando como primer aspecto el concepto drogadicción): el consumo, el abuso y la adicción y en este tercer aspecto consideramos dos procesos fundamentales: los efectos de la tolerancia y el síndrome de supresión.

En la moderna clasificación de los trastornos mentales (Manual Diagnóstico y Estadístico de los Trastornos Mentales-DSM 5) ${ }^{5}$ están enumeradas diez sustancias con efectos posibles de consumo, abuso y adicción (Cuadro 1), incluido el juego de apuestas que comparte características similares con el consumo de otras sustancias.

Estas sustancias pueden ser tan conocidas como la cafeína o el tabaco, o sustancias como la mariguana, el alcohol, la cocaína, algunos me-

Cuadro 1. Diez sustancias incluidas en el DSM 5

\begin{tabular}{l} 
Alcohol \\
Cafeína \\
\hline Cannabis \\
\hline Alucinógenos (fenciclidina y otros) \\
\hline Inhalantes \\
\hline Opiáceos \\
\hline Sedantes \\
\hline Hipnóticos y ansiolíticos \\
\hline Estimulantes (anfetaminas, cocaína y otros) \\
\hline Tabaco
\end{tabular}

Fuente: DSM 5 Capítulo: Trastornos relacionados con sustancias y trastornos adictivos. American Psychiatric Association, Washington 2013. dicamentos, sustancias inhalantes, etcétera, que pueden tener diferente efecto y riesgo de daño, según la dosis y la frecuencia de consumo, pero no debemos olvidar que también depende de la integridad previa del sistema nervioso central del sujeto que las consuma porque con cierta frecuencia podemos ver cómo el consumo único de alguna de estas sustancias desencadena cuadros psiquiátricos de muy difícil control y, en ocasiones, sin posibilidades de recuperación.

Las nuevas sustancias de las que se habla se describen en la mayoría de los casos como drogas de diseño (síntesis química específica que busca un efecto concreto) y que, en el momento actual, se desconoce su efecto a largo plazo, pero que empiezan a mostrar efectos espectaculares (por decirlo de alguna manera) en la conducta de quien las consume. ${ }^{6}$ Cuadro 2

En un país de escaso desarrollo social y económico, las poblaciones de consumidores de estas sustancias no son tan numerosas, dados sus costos; en cambio, el consumo de sustancias como el alcohol, la mariguana, el tabaco y los inhalantes solventes son, por mucho, los que más se consumen; así podemos conocer más de cerca los efectos a corto, mediano y largo plazo de estas sustancias en el cerebro de menores en un proceso de desarrollo activo.

Para el pediatra, la investigación en la evaluación de adolescentes debe incluir preguntas especí-

Cuadro 2. Sustancias de diseño

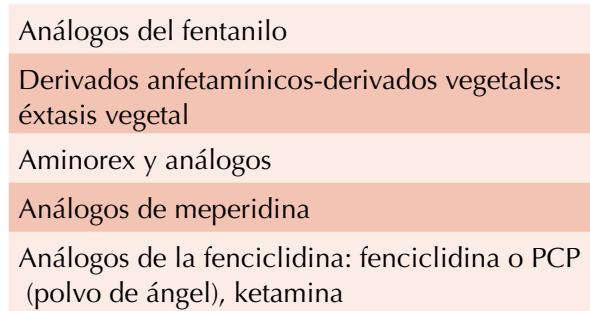

Fuente: Gómez-Ayala AE. Nuevas drogas como opción terapéutica. Farmacia Profesional. 2014;28(3). 
ficas acerca del consumo de sustancias porque en aras de un pudor mal entendido se evitan estas preguntas (cuando no se quiere saber de las posibles respuestas), de la misma manera que es difícil en el interrogatorio hacer preguntas de otros temas, como la ideación suicida en este mismo grupo de pacientes. Un aspecto fundamental es la investigación de la edad de inicio, que ha mostrado correlacionarse con la evolución de un cuadro de abuso de sustancias. ${ }^{7}$

Desde hace varios años se desarrolló una entrevista clinimétrica de tamizaje, que permite detectar el abuso de sustancias, denominada CARLOS, versión en español de la entrevista CRAFFT, ${ }^{8}$ y que el médico pediatra puede aplicar en su consultorio como parte de la evaluación que haga del paciente adolescente. Esta entrevista permite detectar casos que habrá que evaluar más a fondo, pero es un primer acercamiento muy útil para ayudar a estos jóvenes.

La entrevista consta de tres preguntas en la parte $\mathrm{A}$ y seis preguntas en la parte B. Si el joven contesta sí a cualquiera de las tres primeras, se continuará con la parte B y eso permite el tamizaje adecuado, validado ya en nuestro país. ${ }^{9}$ Es posible conseguir esta entrevista a través del Children's Hospital de Boston. ${ }^{10}$

La intoxicación aguda por alcohol es harto conocida, de manera que el médico avezado la puede detectar, pero no es así cuando evaluamos la posibilidad de consumo de mariguana, cocaína o de solventes inhalantes, de manera tal que las conductas de los pacientes en las salas de urgencia, de tipo errático, explosivo, agresivo, de poco control conductual, habla farfullante y estados fluctuantes de la conciencia deben hacer pensar en un síndrome conocido como delirium (estado confusional agudo), ${ }^{11}$ cuya causa deberá buscarse como parte de un protocolo claro que considere opciones etiológicas las infecciosas, las traumáticas $y$, sin duda, las tóxicas, porque se tiende a no considerarlas factor etiológico (sobre todo por la frecuencia con que la familia, los acompañantes o el mismo paciente niegan el consumo de estas sustancias).

Una guía útil para el clínico pediatra en la valoración de casos agudos es la existencia de los siguientes síndromes descritos por Díez-Romero: ${ }^{12}$

Opiáceo. Los consumidores padecen: hipotensión, bradicardia, bradipnea, hipotermia, miosis, coma, choque, síntomas causados por opiáceos y sus derivados, propoxifeno, dextrometorfano y su antídoto es la naloxona.

Sedante-hipnótico. Los consumidores padecen: bradipnea, hipotensión, estupor y coma, causado por benzodiacepinas, barbitúricos, etanol, anticomiciales y neurolépticos.

Alucinógeno. Los consumidores padecen: hipertensión, taquicardia, taquipnea, alucinaciones, diaforesis, desorientación, causados por anfetaminas, cannabinoides, etanol, LSD, incluso antidepresivos inhibidores de la recaptura de serotonina.

Serotoninérgico. Los consumidores padecen: hipertensión, hipotensión, taquicardia, hipertermia, diaforesis, aumento del peristaltismo, hiperreflexia, clono, temblor, agitación, midriasis, causadas por antidepresivos, litio, antieméticos, LSD, éxtasis y cocaína.

Simpaticomimético. Los consumidores padecen: hipertensión, taquicardia, taquipnea, hipertermia, diaforesis, piloerección, hiperreflexia, agitación psicomotora, midriasis, causado por cocaína, anfetaminas, inhibidores de recaptura de noradrenalina, agonistas alfa o beta adrenérgicos.

Cuando se requiere que el médico pediatra determine si el adolescente consume sustancias de abuso, hay que dejar claro que la recomendación es que sea un proceso voluntario del paciente, que no se le engañe obteniendo muestras sin su 
consentimiento (si no es una situación de urgencia) y que, en general, se deberá iniciar por muestras de orina (se pide como antidoping en orina y sirve para detectar alcohol, mariguana, derivados de cocaína, benzodiacepinas, anfetaminas) en la mayor parte de los laboratorios de uso común que si bien no son tan específicas para algunas sustancias, nos dan reporte rápido de la mayor parte de ellas; posteriormente deben hacerse pruebas de concentraciones de esas sustancias en la sangre.

La toxicología en suero (con la utilización de la espectrometría de masa por cromatografía) puede ser útil en situaciones de abuso de múltiples sustancias y de algunos medicamentos psicotrópicos, pero los resultados requieren horas o días, por lo que deben restringirse a situaciones especiales. Siempre debe evaluarse el resultado en función del contexto de la presentación general y no considerarse una herramienta diagnóstica definitiva. ${ }^{13}$

Los estudios a largo plazo muestran que el abuso crónico de estas sustancias (específicamente mariguana) puede desencadenar déficits en la vida adulta, aunque no está claro si sólo la mariguana sería responsable de esta evolución. ${ }^{14}$ Por ello los esfuerzos para detectar tempranamente el consumo abusivo de sustancias puede servir para proteger en general a los jóvenes.

Es importante estar al día de las nuevas sustancias que son consumidas con fines tóxicos, pero sigue siendo más importante que las que conocemos sean exploradas en sus efectos y en sus consecuencias para poder contender adecuadamente y favorecer el manejo terapéutico adecuado, separado de prejuicios y manteniendo el problema dentro de un marco de atención a la salud y al adecuado desarrollo adolescente, estructural y psicosocial.

\section{REFERENCIAS}

1. Miller M, Borges G, Orozco R, Mukamal K, Rimme EB, Benjet $C$, Medina-Mora ME. Exposure to alcohol, drugs and to- bacco and the risk of subsequent suicidality: Findings from the Mexican Adolescent Mental Health Survey. Drug and Alcohol Dependence. 2001:113(2-3);110-117. DOI: https:// doi.org/10.1016/j.drugalcdep.2010.07.016

2. Welty L, Hershfield J, Abram K, Han H, Byck G, Teplin L. Trajectoires of substance use disorder in youth after detention: a 12-year longitudinal study. J Am Acad Child Adolesc Psychiatry. 2017;56(2):140-148.

3. Groenmann A, Jansen T, Oosterlaan J. Childhood psychiatric disorders as risk factor for subsequent substance abuse: a metaanalysis. J Am Acad Child Adolesc Psychiatry. 2017;56(7):556-569.

4. Greenfield B, Sittner K, Forbes M, Walls M, Witbeck L. Conduct disorder and alcohol use disorder trajectories, predictors and outcomes for indigenous youth. J Am Acad Child Adolesc Psychiatry. 2017;56(2):133-139.

5. Diagnostical and Statistical Manual of Mental Diseases 5th edition. (DSM 5) American Psychiatric Association. Washington 2013.

6. Gómez-Ayala A. E. Nuevas drogas como opción terapéutica. Farmacia Profesional. 2014;28(3):23-27.

7. Han B, Compton W, Blanco C, Du Pont R. National trends in substance use and use disorders among youth. J Am Acad Child Adolesc Psychiatry 2017;56(9):747-754.

8. Knight JR, Sherritt L, Shrier LA, Harris SK, Chang G. Validity of the CRAFFT substance abuse screening test among adolescent clinic patients. Archives on Pediatric and Adolescent Medicine. 2002;156(6):607-614. doi: 10.1001/ archpedi.156.6.607.

9. Reyna Barajas GV, Copertari Isaacson LF, González Betanzos F, Padrós Blázquez F. Estudio psicométrico del instrumento CARLOS (CRAFFT) en estudiantes universitarios mexicanos. Evaluar. 2016;16:10-19.

10. C Children's Hospital Boston, 2009. Reproducción exacta del formulario en entornos médicos autorizada por el Center for Adolescent Substance Abuse Research, Children's Hospital Boston, 300 Longwood Ave, Boston, MA 02115, USA, (617) 355-5433, www.ceasar.org

11. Schieveld JNM, Ista E, Knoester H, Molag ML. Pediatric delirium: A practical approach. In Rey JM (ed), IACAPAP e-Textbook of Child and Adolescent Mental Health. Geneva: International Association for Child and Adolescent Psychiatry and Allied Professions 2015.

12. Díez Romero $\mathrm{P}$, Gallego Alonso-Colmenares $\mathrm{M}$, Fernández Herranzy J, Ganzo Pión M. Intoxicaciones agudas por alcohol, otras drogas y fármacos psicoactivos. Medicine. 2015;11(89):5314-23.

13. Riba MB, Ravindranath D. Clinical Manual of Emergency Psychiatry. American Psychiatry Publishing, Inc. 2010. First Edition. Substance-Related Psychiatric Emergencies. Pp. 187-20.

14. Levine A, Clemenza K, Rynn M, Lieberman J. Evidences for the risks and consequences of adolescents cannabis exposure. J Am Acad Child Adolesc Psychiatry. 2017;56(3):214-225. 